\title{
Adverse Drug Effects and Preoperative Medication Factors Related to Perioperative Low-Dose Ketamine Infusions.
}

\author{
Eric S. Schwenk \\ Thomas Jefferson University \\ Stephen F. Goldberg \\ Thomas Jefferson University \\ Ronak D. Patel \\ Thomas Jefferson University \\ Jon Zhou \\ University of California, Davis \\ Poludalas;isandapaditional works at: https://jdc.jefferson.edu/anfp \\ Thomas Jefferson University \\ Part of the Anesthesiology Commons

\section{Let us know how access to this document benefits you} \\ See next page for additional authors
}

\section{Recommended Citation}

Schwenk, Eric S.; Goldberg, Stephen F.; Patel, Ronak D.; Zhou, Jon; Adams, Douglas R.; Baratta, Jaime L.; Viscusi, Eugene R.; and Epstein, Richard H., "Adverse Drug Effects and Preoperative Medication Factors Related to Perioperative Low-Dose Ketamine Infusions." (2016). Department of Anesthesiology Faculty Papers. Paper 33.

https://jdc.jefferson.edu/anfp/33

This Article is brought to you for free and open access by the Jefferson Digital Commons. The Jefferson Digital Commons is a service of Thomas Jefferson University's Center for Teaching and Learning (CTL). The Commons is a showcase for Jefferson books and journals, peer-reviewed scholarly publications, unique historical collections from the University archives, and teaching tools. The Jefferson Digital Commons allows researchers and interested readers anywhere in the world to learn about and keep up to date with Jefferson scholarship. This article has been accepted for inclusion in Department of Anesthesiology Faculty Papers by an authorized administrator of the Jefferson Digital Commons. For more information, please contact: JeffersonDigitalCommons@jefferson.edu. 


\section{Authors}

Eric S. Schwenk, Stephen F. Goldberg, Ronak D. Patel, Jon Zhou, Douglas R. Adams, Jaime L. Baratta, Eugene R. Viscusi, and Richard H. Epstein 


\section{Adverse Drug Effects and Preoperative Medication Factors Related to Perioperative Low-Dose Ketamine Infusions}

\section{Corresponding Author:}

Eric S. Schwenk, MD

Sidney Kimmel Medical College, Thomas Jefferson University

Department of Anesthesiology

Suite 8130, Gibbon Building

111 South $11^{\text {th }}$ Street

Philadelphia, PA 19107

Phone: 215-955-6161

Fax: 215-955-0677

Email: Eric.Schwenk@jefferson.edu

Co-Authors:

Stephen F. Goldberg, MD

Sidney Kimmel Medical College, Thomas Jefferson University

Ronak Patel, MD

Thomas Jefferson University Hospital

Jon Zhou, MD

University of California, Davis

Douglas R. Adams, BS

Sidney Kimmel Medical College, Thomas Jefferson University

Jaime L. Baratta, MD

Sidney Kimmel Medical College, Thomas Jefferson University

Eugene R. Viscusi, MD

Sidney Kimmel Medical College, Thomas Jefferson University

Richard H. Epstein, MD

Sidney Kimmel Medical College, Thomas Jefferson University

\section{Institutional Affiliation of Manuscript:}

Sidney Kimmel Medical College, Thomas Jefferson University

\section{Source of Funding:}

Departmental funding

Portions of this manuscript were presented at the 2013 annual meeting of the American Society of Anesthesiologists and at the 2014 spring meeting of the American Society of Regional Anesthesia and Pain Medicine. 


\section{Conflicts of Interest:}

Eric Schwenk, Stephen Goldberg, Ronak Patel, Jon Zhou, Douglas Adams, Jaime Baratta, and Richard Epstein have no conflicts of interest to declare.

Eugene Viscusi has served as a consultant for AcelRx, Medicines Company, Mallinkrodt, Trevena, Cara Pharmaceuticals, and Astra Zeneca. He has received grant money in the past from AcelRx and Pacira. He has been a paid lecturer for AcelRx, Merck, Salix, and Mallinkrodt. 


\section{Abstract}

2 High-dose opioid administration is associated with significant adverse events.

3 Evidence suggests that low-dose ketamine infusions improve perioperative analgesia over

4 conventional opioid management, but usage is highly variable. Ketamine's adverse drug

5 effects (ADEs) are well known, but their prevalence during low-dose infusions in a

6 clinical setting and how often they lead to infusion discontinuation are unknown. The

7 purposes of this study were threefold: 1) to identify patient factors associated with

8 initiation of ketamine infusions during spine surgery; 2) to identify specific spine

9 procedures in which ketamine has been used most frequently; and 3) to identify ADEs

10 associated with postoperative ketamine infusions and which ADEs most frequently led to

11 discontinuation. Spine surgery was chosen because of its association with moderate to

12 severe pain and a relatively high use of ketamine infusions in this population at our

13 hospital. 


\section{Introduction}

Patients presenting for surgery due to conditions associated with chronic pain

3 frequently are being treated with opioids, often at alarmingly high doses. One study of

4 Medicaid enrollees found that $63.5 \%$ of patients with non-cancer chronic pain had taken

5 an opioid in the prior 12 months, an increase of $18.9 \%$ from five years prior. ${ }^{1}$ Yet despite

6 escalating doses of opioids, patients continue to report that their chronic pain is not well

7 controlled. ${ }^{2}$ At the same time, serious adverse drug effects (ADEs) associated with

8 opioids, including fatal respiratory depression, continue to be a serious concern. ${ }^{3}$ In

9 patients chronically taking opioids, tolerance to respiratory depression is incomplete ${ }^{4}$ and

10 such patients have an increased risk of overdose and death compared to non-opioid

11 users. ${ }^{5}$ Analgesic alternatives to opioids are, therefore, highly desirable.

12 One such alternative during the perioperative period is ketamine, an

$13 \mathrm{~N}$-methyl-D-aspartate (NMDA) receptor antagonist. A role of the NMDA receptor in the

14 development of opioid tolerance was suggested by studies from several decades ago. ${ }^{6,7}$

15 Ketamine is a potent analgesic that does not cause respiratory depression, and may

16 improve postoperative analgesia while reducing opioid consumption. ${ }^{8,9}$ As an additional

17 potential benefit, recent evidence suggests that intravenous (IV) ketamine may decrease

18 the incidence of persistent postsurgical pain (PPSP). ${ }^{10}$ Ketamine infusions at our

19 institution have been used both intraoperatively by the anesthesia team and

20 postoperatively by the acute pain management service (APMS) as part of an

21 opioid-sparing strategy in complex, opioid-tolerant patients.

22 We had three primary objectives of the current study: 1) to identify factors

23 associated with current decisions by anesthesiologists to initiate ketamine during spine 
1 surgery; 2) to identify specific spine procedures in which ketamine has been used most

2 frequently; and 3) to identify ADEs associated with all postoperative ketamine infusions

3 and which ADEs most frequently led to discontinuation. This information is necessary

4 for us to design prospective, randomized clinical trials comparing IV ketamine to placebo

5 in opioid-tolerant patients. Spine surgery was chosen to study in detail because it is

6 typically associated with moderate to severe postoperative pain, over $50 \%$ of our

7 ketamine use has been in spine surgery, and this is a high-volume service at our

8 institution. We retrospectively analyzed the preoperative medications and surgical details

9 of all patients over a three-year period who underwent any type of elective spine surgery

10 and also examined a sample of postoperative patients from all surgical specialties who

11 received ketamine infusions for the presence of ADEs and classified them. 


\section{Methods}

2

4 board without requirement for written patient consent.

12 preoperative medications, and dose and timing data related to ketamine infusions were

13 retrieved from the hospital's anesthesia information management system (Innovian ${ }^{\circledR}$,

14 Dräger, Telford, PA) and from the pharmacy information system database (Pyxis ${ }^{\circledR}$,

15 CareFusion, San Diego, CA). Patients who received intraoperative ketamine boluses but

16 not an infusion were excluded. Data elements analyzed included the date of surgery, age

17 in years, gender, weight, body mass index, American Society of Anesthesiologists (ASA)

18 physical status, scheduled duration of surgery, primary surgical service, and preoperative

19 medications. Preoperative opioids were classified as being taken on a "scheduled" or "as

20 needed" basis (Table 2). Planned procedures (using locally defined, procedure-specific

21 codes) were queried from the operating room case scheduling system $\left(\right.$ ORSOS $^{\circledR}$,

22 McKesson, San Francisco, CA). All planned, elective spine surgeries requiring hospital

23 admission (but not emergencies or cases booked as "add-ons") were included for

24 analysis. The decision to start an intraoperative ketamine infusion at our hospital was 
1 made by the attending anesthesiologist for the case. Data retrieved from the pharmacy

2 information system were aligned with patient anesthesia records to determine if

3 postoperative ketamine infusions had been started intraoperatively or initiated after the

4 patient left the OR.

5 ADE data were retrieved from the daily notes recorded contemporaneously by the

6 Acute Pain Management Service (APMS) nurses on a consecutive sample of 321 patients

7 who received a postoperative ketamine infusion while on the APMS from January 1,

82011 through December 31, 2013. Patients from all surgical subspecialties were included

9 for the ADE analysis, not just those undergoing spine procedures. All APMS nurses had

10 undergone training on the management of ketamine infusions, including the recognition

11 of side effects. No special monitoring, such as telemetry or intensive care, has been

12 required at our institution for patients receiving ketamine. Criteria for discontinuation of

13 ketamine infusions included the patient requesting discontinuation due to ADEs or the

14 patient's primary service requesting discontinuation.

15 Data were extracted and prepared for analysis using SQL Server 2008 R2

16 (Microsoft, Redmond, WA). Odds ratios (OR) were computed using the function

17 oddsRatio in the R mosaic library, Pearson's chi-square test (with Yate's continuity

18 correction), two-group Student t tests (with the Satterthwaite approximation), and local

19 polynomial regression fits using the functions chisq.test, t.test, and loess, respectively, in

20 the R stats library (R v3.2.0, The R Foundation for Statistical Computing, Vienna,

21 Austria). 


\section{Results}

2 Demographics and Preoperative Medications Associated with Ketamine Administration

3 There were 4958 patients who underwent elective spine surgery under general

4 anesthesia during the study interval, 4748 of which were entered into our electronic

5 preoperative anesthesia system and had data available for analysis, and 211 of whom

6 received an intraoperative infusion of ketamine. Among all patients, those receiving

7 ketamine were younger (difference $=-4.4$ years, $95 \%$ CI -2.2 to -6.0 years, $P<10^{-6}$ ), had

8 a higher ASA Physical Status $\left(P<10^{-6}\right)$, and were scheduled for surgeries of longer

9 estimated duration (difference $=72$ minutes, $95 \%$ CI 60 to 84 minutes, $P<10^{-6}$ ), (Table

10 1). There were no significant differences in weight or BMI between the two groups.

11 Males and females were represented equally (difference $=-0.40 \%, 95 \% \mathrm{CI}-3.1 \%$ to

$12 \quad 2.3 \%, P=0.77)$.

13 Medication factors at the time of the preoperative evaluation associated with a

14 greater likelihood of receiving an intraoperative ketamine infusion were taking vs. not

15 taking a scheduled opioid (OR $16.09,95 \%$ CI 11.98 to 21.59), taking any opioid vs. no

16 opioid (OR 10.25, 95\% CI 7.13 to 14.75 ), and taking vs. not taking an anti-depressant

17 (OR 2.69, 95\% CI 2.02 to 3.57) (Table 2). Patients who were taking both a scheduled

18 opioid and an anti-depressant were more likely to receive ketamine than those taking just

19 a scheduled opioid (OR 1.64, 95\% CI 1.11 to 2.46; Table 2).

20 Among the 552 patients who were taking a scheduled opioid at the time of the

21 preoperative evaluation, those receiving a ketamine infusion were younger (difference

$22=-2.8$ years, $95 \%$ CI -0.6 to -5.1 years, $P=0.012)$, had a higher ASA Physical Status $(P$

$23=0.01$ ), and were scheduled for surgeries of longer estimated duration (difference $=49$ 
1 minutes, 95\% CI 32 to 67 minutes, $P<10^{-6}$ ), (Table 3). There were no significant

2 differences in weight or BMI between the two groups. There was a higher proportion of

3 females (difference $=13.3 \%, 95 \%$ CI 4.3 to $22.3 \%, P=0.005$ ).

4

5 Surgical Procedures Associated with Ketamine Administration

6 There were 20 distinct spine procedures (12 primary spine procedures and 8

7 revision spine procedures) identified in the database (Table 4). Of these, there were 10

8 procedures that had $\geq 5 \%$ prevalence of intraoperative ketamine administration. The three

9 most commonly performed of these 10 were posterior thoracic/lumbar fusion $(\mathrm{N}=148$

10 cases), anterior thoracic/lumbar fusion ( $\mathrm{N}=136$ cases $)$, and anterior/posterior cervical

11 fusion $(\mathrm{N}=137$ cases $)$. Specific spine procedures are displayed in Table 4 according to

12 primary or revision status.

13

14 Side Effects of Low-Dose Ketamine Infusions

15 There were $31.8 \%$ of patients who experienced at least one ADE (Table 5). The

16 most frequent ADE was CNS excitation (16.2\%), followed by sedation (9.4\%) and visual

17 disturbances (3.1\%). Some patients experienced more than one ADE (Table 5). Thirty-

18 seven patients (36.3\% of all patients with an ADE) experienced ADEs severe enough to

19 have resulted in discontinuation of the ketamine infusion. The reasons for infusion

20 discontinuation are described in Table 5. Sedation was the ADE most likely to result in

21 ketamine discontinuation. Of the 37 patients whose infusions were discontinued, 35 of

22 them reported resolution of symptoms after the infusion was stopped. Twenty-six patients

23 received benzodiazepines, commonly used for treatment of side effects at our hospital, 
1 while 11 patients did not. To view the TJUH ketamine infusion guidelines, see Appendix 2 A.

3 A postoperative infusion rate above $20 \mathrm{mg} / \mathrm{hr}$ was not associated with an

4 increased chance of having the infusion stopped compared to patients receiving $\leq 20$

$5 \mathrm{mg} / \mathrm{hr}(\mathrm{OR} 0.71,95 \%$ CI 0.34 to 1.5$)$. The chance of discontinuation was also not

6 increased with a threshold of $10 \mathrm{mg} / \mathrm{hr}$ (OR 0.69, 95\% CI 0.32 to 1.5$)$. 


\section{Discussion}

2 In this observational study, we found that patients who received intraoperative

3 ketamine infusions tended to be younger, sicker, and undergoing spine procedures of

4 longer duration (i.e., more complex) than those who did not. Patients who received

5 ketamine infusions were more likely to be taking preoperative opioids, and this increased

6 if patients were taking opioids on a scheduled basis.

7 We found that patients who were most likely to receive ketamine were those

8 undergoing the most complex spine procedures, often involving both an anterior and

9 posterior component or a revision procedure (Table 4). Previous studies examining

10 ketamine in spine surgery have yielded conflicting results regarding postoperative opioid

11 consumption. ${ }^{8,11-13}$ The lack of consistent findings may have resulted, in part, from

12 combining potentially different procedures, listed as "lumbar fusion," "lumbar or

13 thoracolumbar laminectomy and fusion,"12 or "elective spine surgery."13 Our data,

14 however, suggest that anesthesiologists viewed patients undergoing longer, complex

15 procedures differently than less complex cases. For example, one of the most common

16 procedures, primary posterior lumbar fusion, had a low prevalence of ketamine

17 administration, suggesting that providers believed conventional opioid analgesia was

18 adequate. Patients who may benefit most from ketamine should be targeted for future

19 studies examining important long-term outcomes of interest. Those patients undergoing

20 presumably less painful procedures with lower incidence of persistent postsurgical pain,

21 such as primary posterior lumbar fusion or anterior cervical fusion, may be able to be

22 managed with conventional therapy. Consistent with previous studies, ${ }^{9}$ the more complex 
1 and painful spine procedures were the ones most likely to be associated with ketamine

2 administration as a continuous infusion.

3 The apparent increased prevalence of ketamine use in patients taking both anti-

4 depressants and scheduled opioids was not surprising as the link between chronic pain,

5 anti-depressants, opioids, and spine surgery has been described. ${ }^{14}$ Although it is

6 impossible to determine retrospectively if this combined therapy factored into the

7 decision-making process by the anesthesia team, anti-depressant use is a potential

8 confounder that should be controlled for in future studies comparing ketamine to other

9 therapies.

10 Our results also confirm the tolerability of ketamine's ADEs in a clinical setting

11 (Table 5). This agrees with clinical trials in which up to $0.25 \mathrm{mg} / \mathrm{kg} / \mathrm{hr}$ have been tried

12 without major ADEs. ${ }^{13}$ Our observed prevalence of central nervous system ADEs

$13(16.2 \%)$ is similar to the $22 \%$ retrospectively described by Rasmussen. ${ }^{15}$ Thus,

14 consideration of a variable-rate postoperative ketamine infusion in a treatment arm of a

15 randomized clinical trial is reasonable. Our ADE data confirm those from Mayo Clinic,

16 Jacksonville ${ }^{16}$ but go a step further in describing the specific ADEs, their prevalence, and

17 the rate of discontinuation in a daily clinical practice in which the infusion rates are

18 adjusted frequently.

19 Discontinuation of ketamine due to ADEs was unrelated to the maximum dose, a

20 somewhat surprising finding. Several factors may have played a role in this, including

21 ADEs resulting from simultaneous administration of opioids and benzodiazepines, as

22 well as variable patient sensitivity to ketamine, some of which may be related to

23 individual changes at the cellular level. ${ }^{17}$ There may be other unidentified factors as well. 
1 Because 35 out of the 37 patients had resolution of symptoms once the infusions were

2 stopped, this suggests that the side effects were at least partially due to ketamine.

3 Using the data from this observational study, we are currently designing a

4 prospective, randomized trial comparing intra- and postoperative ketamine infusions to

5 placebo in opioid-tolerant patients undergoing complex spine surgery with particular

6 focus on long-term outcomes. Only complex spine procedures that are more likely to

7 result in severe pain will be included (i.e. anterior/posterior procedures or procedures

8 involving two or more spine areas, such as thoracic and lumbar). We will use the

9 frequency of ADEs encountered to guide the process of obtaining informed consent. The

10 ADE data could also be used as a guide for any hospitals considering starting a ketamine

11 service on the general medical floors.

12 Our study may have limited generalizability in that the decision to start an

13 intraoperative ketamine infusion was made at the discretion of the attending

14 anesthesiologist for the case. Thus, our findings may not apply to all practices. Second,

15 some patients may have been inappropriately placed in the "scheduled" opioid group

16 rather than the "as needed" group or vice versa due to documentation issues or patients

17 misrepresenting their opioid use.

18 In conclusion, we confirmed that postoperative ketamine infusions may be given

19 safely on general medical floors without special monitoring or intensive care, and

20 intraoperative infusions tend to be started for patients taking opioids, especially

21 scheduled opioids. Our data provide guidance both for hospitals considering the use of

22 ketamine infusions and for the design of future prospective, randomized clinical trials

23 looking at long-term benefits of ketamine or its ADEs. 


\section{References}

1. Sullivan MD, Edlund MJ, Fan M, DeVries A, Braden JB, Martin BC. Trends in use of opioids for non-cancer pain conditions from 2000-2005 in Commercial and Medicaid insurance plans: The TROUP study. Pain 2008;138:440-449

2. Fredheim OMS, Mahic M, Shurtveit S, Dale O, Romundstad P, Borchgrevink PC. Chronic pain and use of opioids: A population-based pharmacoepidemiological study from the Norwegian Prescription Database and the Nord-Trondelag Health Study. Pain 2014;155:1213-1221

3. Benyamin R, Trescot AM, Datta S, et al. Opioid Complications and Side Effects. Pain Physician 2008;11:S105-S120

4. White JM, Irvine RJ. Mechanisms of fatal opioid overdose. Addiction 1999;94:961972

5. Dunn KM, Saunders KW, Rutter CM, et al. Opioid Prescriptions for Chronic Pain and Overdose. Ann Int Med 2010;152:85-92

6. Trujillo KA, Akil H. Inhibition of Morphine Tolerance and Dependence by the NMDA Antagonist MK-801. Science 1991;251:85-87

7. Elliott K, Minami N, Kolesnikov YA, Pasternak GW, Inturrisi CE. The NMDA receptor antagonists, LY274614 and MK-801, and the nitric oxide synthase inhibitor, NG-nitro-L-arginine, attenuate analgesic tolerance to the mu-opioid morphine but not to kappa opioids. Pain 1994;56:69-75 
8. Loftus RW, Yeager MP, Clark JA, et al. Intraoperative Ketamine Reduces Perioperative Opiate Consumption in Opiate-dependent Patients with Chronic Back Pain Undergoing Back Surgery. Anesthesiology 2010;113:639-646

9. Laskowski K, Stirling A, McKay WP, Lim HJ. A systematic review of intravenous ketamine for postoperative analgesia. Can J Anesth 2011;58:911-923

10. McNicol ED, Schumann R, Haroutounian S. A systematic review and meta-analysis of ketamine for the prevention of persistent post-surgical pain. Acta Anaesthesiol Scand 2014;58:1199-1213

11. Urban MK, Ya Deau JT, Wukovits B, Lipnitsky JY. Ketamine as an Adjunct to Postoperative Pain Management in Opioid Tolerant Patients After Spinal Fusions: A Prospective Randomized Trial. HSSJ 2008;4:62-65

12. Subramaniam K, Akhouri V, Glazer PA, et al. Intra- and Postoperative Very Low Dose Intravenous Ketamine Infusion Does Not Increase Pain Relief after Major Spine Surgery in Patients with Preoperative Narcotic Analgesic Intake. Pain Med 2011;12:1276-1283

13. Garg N, Panda NB, Gandhi KA, et al. Comparison of Small Dose Ketamine and Dexmedetomidine Infusion for Postoperative Analgesia in Spine Surgery - A Prospective Randomized Double-blind Placebo Controlled Study. J Neurosurg Anesthesiol 2015;May 14 epub ahead of print

14. Walid MS, Zaytseva NV. Prevalence of mood-altering and opioid medication use among spine surgery candidates and relationship with hospital cost. J Clin Neurosci 2010;17:597-600 
15. Rasmussen KG. Psychiatric side effects of ketamine in hospitalized medical patients administered subanesthetic doses for pain control. Acta Neuropsychiatr 2014;26:230233

16. Porter SB, McClain RL, Howe BL, et al. Perioperative Ketamine for Acute Postoperative Analgesia: The Mayo Clinic-Florida Experience. J Perianesth Nurs 2015;30:189-195

17. Villasenor A, Ramamoorthy A, Silva dos Santos M, et al. A pilot study of plasma metabolomic patterns from patients treated with ketamine for bipolar depression: evidence for a response-related difference in mitochondrial networks. Br J Pharmacol 2014;171:2230-2242. 